\title{
Attenuated Chimpanzee Adenovirus 5T4 Vaccine
}

National Cancer Institute

\section{Source}

National Cancer Institute. Attenuated Chimpanzee Adenovirus 5T4 Vaccine. NCI

Thesaurus. Code C121544.

A cancer vaccine comprised of a recombinant, attenuated, replication-defective simian adenovirus vector (ChAdOx1) encoding the human 5T 4 fetal oncoprotein (ChAdOx1.5T 4), with potential immuno-activating and antineoplastic activities. Upon administration of the recombinant attenuated chimpanzee adenovirus 5T 4 vaccine, the viral vector expresses 5T 4 and stimulates the host immune system to mount a cytotoxic T-lymphocyte (CTL) response against tumor cells expressing 5T4, which results in tumor cell lysis. 5T 4, a transmembrane glycoprotein, is overexpressed by a variety of cancer cell types; its expression is correlated with increased invasiveness. 